Paper

\title{
Statistical test of quasiperiodicity in the presence of dynamical noise
}

\author{
Natsuhiro Ichinose ${ }^{1 a)}$, Yoshihiko Horio ${ }^{2}$, \\ and Masahiro Ogawa* 3 \\ ${ }^{1}$ Department of Intelligence Science and Technology, Graduate School of \\ Informatics, Kyoto University, \\ Yoshida-Honmachi, Sakyo-ku, Kyoto 606-8501, Japan \\ ${ }^{2}$ Research Institute of Electrical Communication, Tohoku University, \\ 2-1-1 Katahira, Aoba-ku, Sendai, Miyagi 980-8577, Japan \\ ${ }^{3}$ Graduate School of Engineering, Tokyo Denki University, \\ 5 Senju Asahi-cho, Adachi-ku, Tokyo 120-8551, Japan \\ a)ichinose@i.kyoto-u.ac.jp
}

Received July 13, 2017; Revised November 22, 2017; Published April 1, 2018

\begin{abstract}
We propose a statistical method to test whether time series data are quasiperiodic or periodic. A time series is defined to be quasiperiodic if its generating map is topologically conjugate to an irrational rotation. We present an algorithm to estimate the conjugacy map from time series data. We also show that a noisy irrational rotation is equivalent to a random walk model. Since there are general tests for a random walk, we can statistically evaluate quasiperiodicity of a time series by using the estimated conjugacy map. The proposed method is validated by asymmetric chaotic neural networks controlled to be quasiperiodic.
\end{abstract}

Key Words: quasiperiodicity, irrational rotation, dynamical noise, statistical test, chaotic neural network

\section{Introduction}

Periodicity, quasiperiodicity and chaos are commonly observed behaviors in dynamical systems [1]. Our interest is to determine the functional meanings of each behavior in neural systems. There are many applications of periodicity and chaos to neural systems. For example, periodicity (including fixed points) has been applied to associative memory because of its ability to reduce noise $[2-5]$. Chaos has been applied to solution searches in optimization problems because of its variety of orbits [6-9]. On the other hand, although the existence of quasiperiodicity has been shown at the single-neuron level [10], its application is not necessarily complete. Thus, aiming towards the application of quasiperiodicity to neural systems, we have proposed several methods to control neural networks to be quasiperiodic [11-

* M. Ogawa is currently with Electronics System Design Div. 1, Toyota Compact Car Company, Toyota-cho, Toyota, Aichi 471-8572, Japan. 
13], along with their implemented circuits $[14,15]$. The fundamental issue of these works is that it is difficult to conclude whether a time series obtained from the system is quasiperiodic or not, especially in real-world systems such as implemented circuits.

In this work, we are focused on the discrimination between periodicity and quasiperiodicity. Lyapunov exponents are the general tool to detect their differences: we can conclude that the system is periodic if the largest Lyapunov exponent is negative, and quasiperiodic if it is zero. Lyapunov exponents can be calculated from an observed time series even if we have no detailed information of the system [16]. Therefore, we can apply the method of Lyapunov exponents to real-world systems in which we cannot know the detailed internal parameters or structures. However, a critical problem is that quasiperiodicity is detected only at the single point where the largest Lyapunov exponent is zero, in contrast to periodicity. This problem arises because dynamical noise is inevitable in realworld systems. As a result, the largest Lyapunov exponent is not fixed to the single point and can be fluctuated. In this work, therefore, we propose a statistical method to test whether the obtained fluctuation is derived from a quasiperiodic system in the presence of dynamical noise or not.

The main idea of our method is to utilize the equivalence between a quasiperiodic system in the presence of dynamical noise and a random walk model. The random walk is used for modelling a time series of real-world systems such as financial markets [17]. In the random walk, since the internal state does not decay in time, the effect of noise is cumulatively additive into the system. It is known that such a time series is non-stationary [18]. On the other hand, since the largest Lyapunov exponent of the quasiperiodic system is zero, its orbit is not expanded or shrunk for a perturbation. Therefore, in the same way as the random walk, the effect of noise is also cumulative in the quasiperiodic system. The general tests for the random walk have been proposed [19]. Using these methods, we can statistically test whether a time series is a random walk or not. Since this is equivalent to testing whether the largest Lyapunov exponent is zero or not, the statistical methods of the random walk can be directly applied to the detection of quasiperiodicity. It should be noted that dynamical noise in our method is not a subject to be avoided and serves as an effective probe to detect quasiperiodicity.

In Sec. 2, we introduce topological conjugacy of a quasiperiodic system to an irrational rotation, and show a simple algorithm to find the conjugacy map from a time series. In the presence of dynamical noise, we show that a noisy irrational rotation is equivalent to a random walk model and its statistical test is feasible. In Sec. 3, as an example of quasiperiodicity in neural systems, we introduce a method to control asymmetric chaotic neural networks to be quasiperiodic. Using this system, we evaluate dependence on noise intensity in our method.

\section{Methods}

\subsection{Quasiperiodicity and irrational rotation}

In this work, we are focused on discrete-time dynamics on an invariant closed curve (1-torus). If we consider flow on a 2-torus, we use the Poincaré section to construct a return map [20]. In the case of a higher-order torus, the Poincaré slice method helps us to obtain the invariant closed curve [21].

Let $f$ be an orientation-preserving map of the circle $\boldsymbol{S}^{1}=\boldsymbol{R} / \boldsymbol{Z}$, generating an orbit:

$$
\phi_{n+1}=f\left(\phi_{n}\right),
$$

where $\phi_{n} \in \boldsymbol{S}^{1}$ is the phase variable at the time $n$. Let $F: \boldsymbol{R} \rightarrow \boldsymbol{R}$ be a lift of $f$. The lift is used to analyze the map on $\boldsymbol{R}$ that is originally defined on $\boldsymbol{S}^{1}$. The following conditions hold in $F$ : $F(\phi+1)=F(\phi)+1$ and $f(\phi)=F(\phi)(\bmod 1)$. The condition in which $f$ is orientation-preserving implies that $F$ is strictly increasing. The rotation number $\omega$ is an important invariant of quasiperiodic orbits [22], and defined as follows by using $F$,

$$
\omega=\lim _{n \rightarrow \infty} \frac{F^{n}\left(\phi_{0}\right)-\phi_{0}}{n} .
$$

Poincaré proved that the limit $\omega$ is determined independently of the initial state $\phi_{0}$. Intuitively, $\omega$ corresponds to an average phase difference for an iteration of the phase variable. If the dynamics of 
$f$ are quasiperiodic, $\omega$ is an irrational number. It is proved that $f$ with irrational rotation number $\omega$ is topologically conjugate to an irrational rotation [23], defined by,

$$
\theta_{n+1}=T_{\omega}\left(\theta_{n}\right)=\theta_{n}+\omega(\bmod 1)
$$

where $\theta_{n} \in \boldsymbol{S}^{1}$. This implies that there exists a unique conjugacy map $h$ such that $h \circ f=T_{\omega} \circ h$.

In this work, we discuss whether the given time series is quasiperiodic, rather than mapping $f$ itself. In general, we obtain the time series as a vector series of $\boldsymbol{R}^{N}$ where $N$ is the dimension of observations. If the time series is quasiperiodic, there exists its attractor on the invariant closed curve. Therefore, the time series of $\boldsymbol{R}^{N}$ can be transformed into that of $\boldsymbol{S}^{1}$. Then, we define that the transformed phase series $\left\{\phi_{n}\right\}$ of $\boldsymbol{S}^{1}$ is quasiperiodic if there exists the conjugacy map $h$ between the underlying map $f$ and the irrational rotation, i.e., $\theta_{n}=h\left(\phi_{n}\right)$. There are two problems to show the existence of $h$. The first problem is how to construct $h$ from the given time series. Since $f$ is unknown, we cannot directly construct $h$ from $f$. The second problem is how to decide whether $h\left(\phi_{n}\right)$ is actually the irrational rotation. As mentioned in the introduction, dynamical noise plays an important role in this problem. We discuss these problems in the following sections.

\subsection{Simple algorithm to find conjugacy map}

We consider the construction of the phase series from the observed time series. We assume that we can choose the two-dimensional time series properly representing the invariant closed curve from the observed time series of $\boldsymbol{R}^{N}$. Then, as shown in Fig. 1, we obtain the invariant closed curve in a two-dimensional plane. We choose origin $O$ and center $C$ on and inside the invariant closed curve, respectively. Each point of the invariant closed curve is transformed as an angle from the segment $C O$ as shown in Fig. 1(a).

(a)

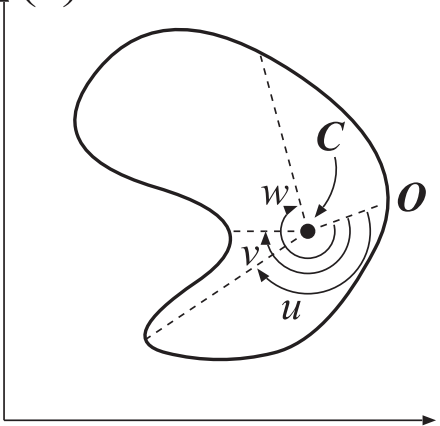

(b)

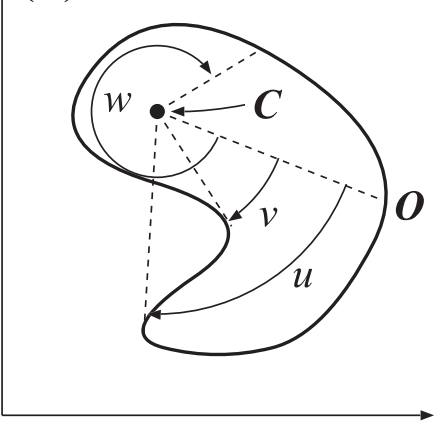

Fig. 1. Transformation of time series on invariant closed curve to phase. $C$ and $O$ are the center and origin of the phase, respectively. By the choice of $C$, the consistency of phase with the orientation of points on the invariant closed curve is determined. (a) Consistent case: $u<v<w$. (b) Inconsistent case: $v<u<w$.

The choice of origin $O$ is arbitrary. However, center $C$ must be chosen such that the transformed phase is orientation-preserving. In Fig. 1(b), although the order of points on the invariant closed curve is $u<v<w$, the transformed phases are inconsistent with that order, i.e. $v<u<w$. In this case, even if the underlying map $f$ is orientation-preserving, we cannot obtain the orientationpreserving phase series. Therefore, we choose $C$ such that the segment between $C$ and each point of the invariant closed curve does not intersect with the invariant closed curve except at the endpoints. If the observed time series is one-dimensional $(N=1)$ or there are some self-intersections of the invariant closed curve, we can use delay coordinates to obtain the higher-dimensional time series and resolve the intersections [24].

Using the phase series $\left\{\phi_{n}\right\}$, we construct the conjugacy map $h$. To find $h$, an invariant measure of $f$ plays an important role. Let $\mu$ be the invariant measure of $f$. In general, $\mu$ is not uniform because the phase difference changes for each iteration. On the other hand, since the irrational rotation has 
a fixed phase difference $\omega$, its invariant measure is uniform. Therefore, $h$ is the map that transforms $\mu$ to be uniform. In fact, the conjugacy map $h$ is represented as follows by using $\mu$ [25],

$$
h(\phi)=\int_{0}^{\phi} \mu(\nu) d \nu
$$

Since $f$ is unknown, $\mu$ is also unknown. However, we can construct an empirical distribution using $\left\{\phi_{n}\right\}$ instead of $\mu$. Therefore, we can approximately represent the conjugacy map $h$ as follows,

$$
h(\phi) \approx \int_{0}^{\phi} \sum_{n=1}^{M} \delta\left(\phi_{n}-\nu\right) d \nu,
$$

where $M$ is the number of data and $\delta$ is the delta function.

Equation (5) for each phase $\phi_{n}$ is calculated by the following procedure. First, we sort the time series $\left\{\phi_{1}, \phi_{2}, \ldots, \phi_{M}\right\}$ in ascending order. Then, we obtain the sorted series $\left\{\phi_{s(1)}, \phi_{s(2)}, \ldots, \phi_{s(M)}\right\}$ (i.e. $\phi_{s(n)} \leq \phi_{s(n+1)}$ for all $n<M$ ). The conjugacy map of each phase is calculated as follows,

$$
\theta_{s(n)}=h\left(\phi_{s(n)}\right) \approx \frac{n-1}{M} .
$$

Then, we obtain the sorted series of the irrational rotation $\left\{\theta_{s(1)}, \theta_{s(2)}, \ldots, \theta_{s(M)}\right\}$. We reversely sort this series in time order and obtain the time series of the irrational rotation: $\left\{h\left(\phi_{n}\right)\right\}=$ $\left\{\theta_{1}, \theta_{2}, \ldots, \theta_{M}\right\}$.

\subsection{Noisy irrational rotation as random walk model}

We can obtain the time series of the irrational rotation using the conjugacy map $h$ constructed in the previous section if the observed time series is quasiperiodic. However, even if the observed time series is periodic, the transformation can be constructed in the same way. Therefore, to show the existence of $h$, we must test whether the transformed time series is the irrational rotation. For this statistical test, we show the equivalence between the irrational rotation in the presence of dynamical noise and a random walk model.

We consider the following system of the orientation-preserving map $f$ in the presence of (additive) dynamical noise:

$$
\phi_{n+1}=f\left(\phi_{n}\right)+\epsilon_{n},
$$

where $\epsilon_{n}$ is white noise. In the condition of small $\epsilon_{n}$, we transform the system into the irrational rotation by using a Taylor series of $h$ :

$$
h\left(f\left(\phi_{n}\right)+\epsilon_{n}\right)=\theta_{n}+\omega+\left.\frac{d h}{d \phi}\right|_{\phi=f\left(\phi_{n}\right)} \epsilon_{n}+O\left(\epsilon_{n}^{2}\right)(\bmod 1),
$$

where $h\left(f\left(\phi_{n}\right)\right)=\theta_{n}+\omega(\bmod 1), O$ is Landau notation that denotes the error terms, and $d h / d \phi$ is the quasiperiodic version of the phase response curve [11]. The phase response curve is used to analyze the effect of a perturbation in continuous-time periodic orbits [26]. We have extended this method to quasiperiodic systems [11]. The phase response curve $d h / d \phi$ is dependent on the phase $\phi_{n}$. In this case, the noise term is also dependent on the state of the system. However, it is noted that the transformation method in the previous section makes the invariant measure uniform even in the presence of dynamical noise. Therefore, we assume that the phase response curve is also uniform: $d h / d \phi=c$ (constant). Then, we approximately represent the irrational rotation in the presence of dynamical noise as follows,

$$
\theta_{n+1}=\theta_{n}+\omega+\epsilon_{n}(\bmod 1),
$$

where we rewrite $c \epsilon_{n}$ as $\epsilon_{n}$. Since Eq. (9) is defined in $\boldsymbol{S}^{1}$, we use the lift system to simplify its arithmetic manipulation. Let $\Theta_{n} \in \boldsymbol{R}$ be the variable of the lift of the irrational rotation. The lift system is represented as follows,

$$
\Theta_{n+1}=\Theta_{n}+\omega+\epsilon_{n}
$$


It is noted that " $(\bmod 1)$ " in Eq. (9) vanishes in Eq. (10), and the other terms are identical. Since $\theta_{n}=\Theta_{n}(\bmod 1)$, the lift system $(10)$ preserves the properties of the original system (9).

It is recognizable that the lift system (10) is similar to an autoregressive model:

$$
r_{n+1}=\varphi r_{n}+\tau+\epsilon_{n},
$$

where $\varphi$ and $\tau$ are the parameters of the model, and $r_{n} \in \boldsymbol{R}$. If $|\varphi|<1$, the autoregressive model is a stationary process. If $\varphi=1$, the autoregressive model is a random walk (with trend). The random walk model is defined by the following process $R_{n}$ for $\left\{X_{1}, X_{2}, \ldots\right\}$,

$$
R_{n}=\sum_{m=1}^{n} X_{m},
$$

where $X_{m}$ is an independent and identically distributed random variable. If $X_{m}$ is normally distributed with variance $\sigma^{2}$, the variance of $R_{n}$ is dependent on time $n$ : $\operatorname{Var}\left(R_{n}\right)=n \sigma^{2}$. Therefore, the process of the random walk is non-stationary. If $\varphi=1$, the autoregressive model (11) is solved as follows,

$$
r_{n}=r_{0}+n \tau+\sum_{m=0}^{n} \epsilon_{m},
$$

where $r_{0}+n \tau$ is referred to as the trend. The summation term of Eq. (13) is equivalent to the random walk (12). Therefore, if $\varphi=1$ and $\tau=\omega$, the lift system of the irrational rotation (10) is equivalent to the autoregressive model (13), and hence, the random walk with trend.

\subsection{Statistical test}

In econometrics, several statistical methods have been proposed to test whether a time series is a random walk or not. This is because a spurious regression of such a time series occurs due to the nonstationarity of the random walk [18]. In such a case, a spurious correlation is detected even between independent time series. Since financial data tend to be random walks, the statistical test of the random walk is important in econometrics. The Dicky-Fuller test is one such statistical method [19].

In the Dicky-Fuller test (and the other methods), the null hypothesis is that the time series is a random walk, i.e., $\varphi=1$ in the autoregressive model (11). The alternative hypothesis is that $\varphi<1$. If the $p$-value is less than a significance level, the null hypothesis is rejected and we conclude that $\varphi<1$. Therefore, it should be noted that we accept that $\varphi=1$ if the $p$-value is greater than the significance level.

The Dicky-Fuller test provides the statistical criterion for the discrimination between quasiperiodicity and periodicity. However, our method cannot be used for discriminating chaos. We discriminate quasiperiodicity and periodicity by whether $\varphi=1$ or $\varphi<1$. Analogously, it is expected that the case of $\varphi>1$ implies chaos. The Dicky-Fuller test is also able to cope with such an exponential growth case. However, since we use the phase transformation of the orbit, a phase difference cannot exceed one period of the invariant closed curve. As a result, since the exponential growth (i.e. $\varphi>1$ ) is not realized in the phase series, the detection of chaos is difficult for our method and remains for future work.

In results, we adopt the augmented Dicky-Fuller test [27]. In the augmented test, we can use a higher-order autoregressive model than Eq. (11). The time series has a temporal correlation due to an inhomogeneous phase response curve. Although we assume that the phase response curve is uniform, it is actually dependent on the phase, hence the noise in the irrational rotation has a correlation in time. Since the augmented Dicky-Fuller test cancels this correlation by using the higher-order autoregressive model, the value of $\varphi$ can be correctly estimated.

\subsection{Algorithm summary}

We summarize the algorithm to detect quasiperiodicity explained in the previous sections. Suppose that the two-dimensional time series is given as analyzing data: $\left\{\left(x_{n}, y_{n}\right)\right\}$. Let origin $O$ of the phase be the direction of the $y$-axis. The algorithm is performed by the following steps. 
1. Choose center $C=\left(c_{x}, c_{y}\right)$ inside the invariant closed curve of $\left\{\left(x_{n}, y_{n}\right)\right\}$.

2. Convert $\left(x_{n}, y_{n}\right)$ to phase $\phi_{n}$ :

$$
\phi_{n}=\frac{\pi+\operatorname{atan} 2\left(y_{n}-c_{y}, x_{n}-c_{x}\right)}{2 \pi},
$$

where atan2 is the standard programming function, arctangent, defined in $[-\pi, \pi]$.

3. Sort $\left\{\phi_{1}, \phi_{2}, \ldots, \phi_{M}\right\}$ in ascending order and obtain $\left\{\phi_{s(1)}, \phi_{s(2)}, \ldots, \phi_{s(M)}\right\}$ where $\phi_{s(n)} \leq$ $\phi_{s(n+1)}$ for all $n<M$.

4. Convert $\phi_{s(n)}$ to the irrational rotation $\theta_{s(n)}: \theta_{s(n)}=\frac{n-1}{M}$.

5. Reversely sort $\left\{\theta_{s(1)}, \theta_{s(2)}, \ldots, \theta_{s(M)}\right\}$ in time order and obtain $\left\{\theta_{1}, \theta_{2}, \ldots, \theta_{M}\right\}$.

6. Convert $\theta_{n}$ to the lift $\Theta_{n}\left(\theta_{0}=0, \Theta_{0}=0\right)$ :

$$
\Theta_{n}=\Theta_{n-1}+\left\{\begin{array}{ll}
\theta_{n}+1-\theta_{n-1} & \text { if } \theta_{n}<\theta_{n-1} \\
\theta_{n}-\theta_{n-1} & \text { otherwise }
\end{array} .\right.
$$

7. Apply the augmented Dicky-Fuller test to $\left\{\Theta_{n}\right\}$.

8. If the $p$-value is greater than a significance level, we accept that $\left\{\left(x_{n}, y_{n}\right)\right\}$ is quasiperiodic.

In the following results, we use R function "adf.test" (augmented Dicky-Fuller test) to test the data [28].

\section{Results and discussion}

\subsection{Control of quasiperiodicity in asymmetric chaotic neural networks}

To evaluate our method, we use the chaotic neural networks controlled to be quasiperiodic by the pole assignment method, which is widely used in control theory [12,29]. We consider the following chaotic neural network with asymmetric connections:

$$
\begin{aligned}
& x_{1}(n+1)=k x_{1}(n)-(\alpha-\beta) y_{1}(n)+a-\frac{\beta+d}{2} y_{3}(n)-\frac{\beta-d}{2} y_{2}(n), \\
& x_{2}(n+1)=k x_{2}(n)-(\alpha-\beta) y_{2}(n)+a-\frac{\beta+d}{2} y_{1}(n)-\frac{\beta-d}{2} y_{3}(n), \\
& x_{3}(n+1)=k x_{3}(n)-(\alpha-\beta) y_{3}(n)+a-\frac{\beta+d}{2} y_{2}(n)-\frac{\beta-d}{2} y_{1}(n),
\end{aligned}
$$

where $k$ is the decay parameter, $\alpha$ is the parameter of refractoriness, $\beta$ is the connection weight, $a$ is the bias used as the bifurcation parameter, $d$ is the asymmetric parameter, $x_{i}(n)$ is the internal state of the $i$-th neuron at the time $n$, and $y_{i}(n)$ is the output of the $i$-th neuron with parameter $\epsilon$ :

$$
y_{i}(n)=f\left(x_{i}(n)\right), \quad f(x)=\frac{1}{1+e^{-x / \epsilon}} .
$$

This system has the synchronous solutions $x_{1}(n)=x_{2}(n)=x_{3}(n)$ equivalent to the solutions of the single neuron:

$$
x_{1}(n+1)=k x_{1}(n)-\alpha y_{1}(n)+a .
$$

Let $x^{*}$ be the synchronous fixed point: $x^{*}=x_{1}(n)=x_{2}(n)=x_{3}(n)$. The Jacobian matrix of $x^{*}$ has a real eigenvalue $\lambda_{1}$ and a pair of complex eigenvalues $\lambda_{2,3}$ :

$$
\lambda_{1}=k-\alpha \gamma, \quad \lambda_{2,3}=k-\left(\alpha-\frac{3}{2} \beta\right) \gamma \pm i \frac{\sqrt{3}}{2} d \gamma,
$$

where $\gamma=f^{\prime}\left(x^{*}\right)$. If the complex eigenvalues are destabilized, i.e. $\left|\lambda_{2,3}\right|>1$, the Neimark-Sacker bifurcation occurs and a quasiperiodic orbit is generated. Since the complex eigenvalues do not exist 
if $d=0$, the asymmetricity of connections is important for quasiperiodic orbits generated around $x^{*}$. However, if $\lambda_{1}<-1$, the period-doubling bifurcation occurs and the quasiperiodic orbit can become unstable. Therefore, we stabilize the orbit by stabilizing $\lambda_{1}$.

We consider the following feedback system:

$$
\boldsymbol{x}(n+1)=\boldsymbol{F}(\boldsymbol{x}(n))+g \boldsymbol{u}(n),
$$

where $\boldsymbol{x}(n)=\left(x_{1}(n), x_{2}(n), x_{3}(n)\right)^{T}, \boldsymbol{F}$ is the chaotic neural network of Eq. (16), (17), and (18), $g$ is the feedback gain, and $\boldsymbol{u}(n)$ is the feedback input represented as follows,

$$
\boldsymbol{u}(n)=(u(n), u(n), u(n))^{T}, \quad u(n)=y_{1}(n)+y_{2}(n)+y_{3}(n)-3 f\left(x^{*}\right) .
$$

The pole assignment method can design the value of $g$ to assign $\lambda_{1}$ to an arbitrary value without changing $\lambda_{2,3}$ (see [12] for details). If we assign $\lambda_{1}$ to a real eigenvalue $\lambda, g$ is represented as follows,

$$
g=\frac{\lambda-k+\alpha \gamma}{3 \gamma}
$$

If $|\lambda|<1$, the period-doubling bifurcation does not occur at $x^{*}$ and we can observe the quasiperiodic orbit. It should be noted that the quasiperiodic orbit itself is not necessarily stabilized. Since $\lambda_{2,3}$ changes with the bifurcation parameter $a$, we can also observe periodic orbits by phase locking.

In the following results, for simplicity, we use $\gamma$ as the bifurcation parameter instead of $a$. This is because we can obtain $x^{*}$ and $a$ from $\gamma$, whereas we cannot analytically obtain $x^{*}$ from $a$.

\subsection{Evaluation of dependence on noise intensity}

To evaluate the effect of dynamical noise, we use the following system based on Eq. (22):

$$
\boldsymbol{x}(n+1)=\boldsymbol{F}(\boldsymbol{x}(n))+g \boldsymbol{u}(n)+\sigma \boldsymbol{\epsilon}(n),
$$

where $\sigma$ is the intensity of dynamical noise, $\boldsymbol{\epsilon}(n)=\left(\epsilon_{1}(n), \epsilon_{2}(n), \epsilon_{3}(n)\right)^{T}$ is the vector of random variables, and each variable is normally distributed with zero mean and unit variance. We show the examples of a quasiperiodic orbit in Fig. 2(a), and a periodic orbit in Fig. 2(b) in the noiseless case $(\sigma=0)$. The periodic orbit has 15 periodic points. We choose $\gamma$ of the periodic and quasiperiodic orbits such that their values are close to each other. Then, the two orbits are affected by each other in their properties. In Fig. 3, we show the empirical invariant measure $\mu$ corresponding to the quasiperiodic orbit of Fig. 2(a). In the invariant measure, there are 15 local maxima which correspond to the 15 periodic points, implying that the orbit is biased at each point. In fact, the rotation number of the quasiperiodic orbit is close to 15 periods: $\omega=1.3328 \ldots \approx 2 / 15$. Therefore, the two orbits are difficult to discriminate from each other in general.

(a)

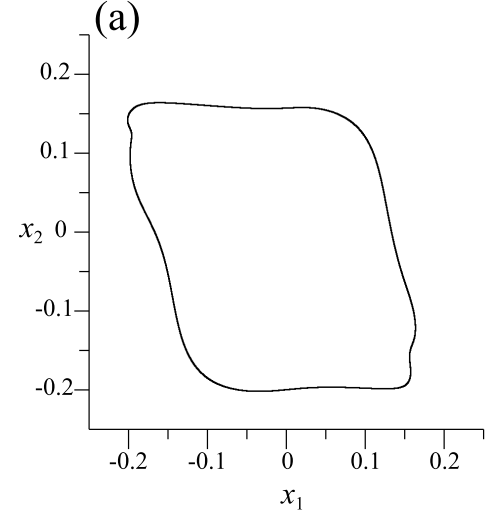

(b)

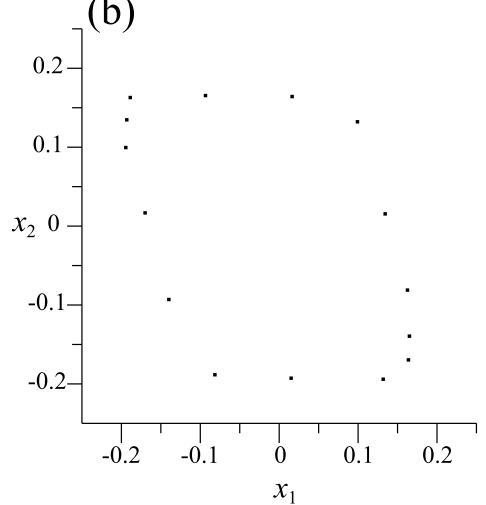

Fig. 2. Orbits of chaotic neural networks $(\sigma=0)$. (a) Quasiperiodic orbit $(\gamma=4.98)$. (b) Periodic orbit $(\gamma=4.9895)$. The largest Lyapunov exponents are $-7.40 \times 10^{-6}$ for (a), and -0.00781 for (b). The other parameters are fixed at $k=0.7, \alpha=1, \beta=2 / 3, d=0.3, \epsilon=0.05$, and $\lambda=-0.9$. 


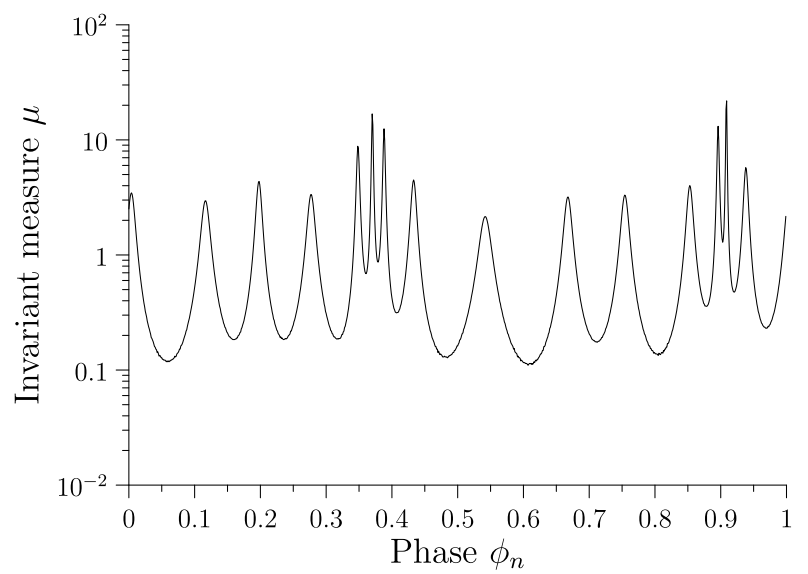

Fig. 3. Empirical invariant measure $\mu$ of Fig. 2(a). The center of phase is $C=(0,0)$.

(a)

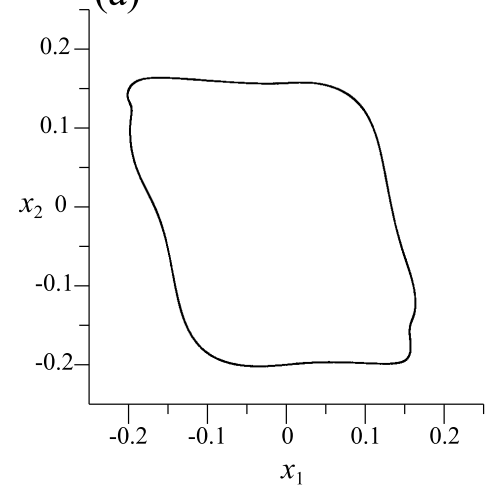

(b)

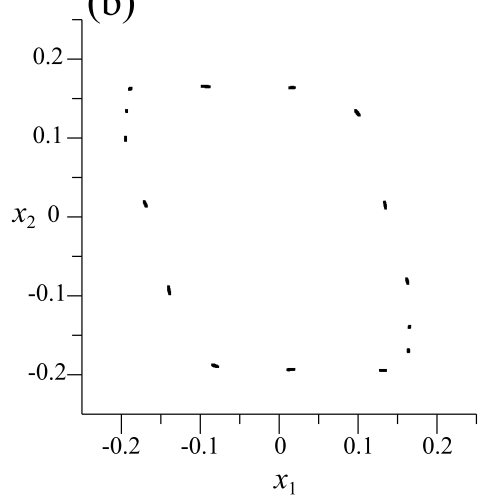

Fig. 4. Orbits of chaotic neural networks in the low-noise case $(\sigma=0.0001)$. (a) Quasiperiodic orbit $(\gamma=4.98)$. (b) Periodic orbit $(\gamma=4.9895)$. The other parameters are fixed at $k=0.7, \alpha=1, \beta=2 / 3, d=0.3, \epsilon=0.05$, and $\lambda=-0.9$.

(a)

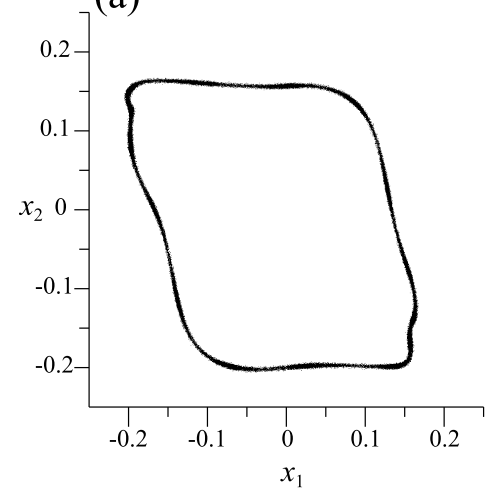

(b)

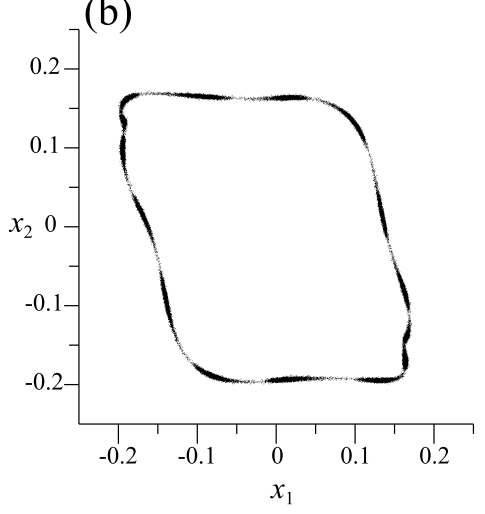

Fig. 5. Orbits of chaotic neural networks in the medium-noise case $(\sigma=$ 0.001). (a) Quasiperiodic orbit $(\gamma=4.98)$. (b) Periodic orbit $(\gamma=4.9895)$. The other parameters are fixed at $k=0.7, \alpha=1, \beta=2 / 3, d=0.3, \epsilon=0.05$, and $\lambda=-0.9$.

To evaluate the dependence of our method on noise intensity, we show the quasiperiodic and periodic orbits in the low-noise case ( $\sigma=0.0001$, Fig. 4$)$, medium-noise case $(\sigma=0.001$, Fig. 5$)$, and high-noise case $(\sigma=0.01$, Fig. 6$)$. Although we can recognize that the quasiperiodic orbit is different from the periodic orbit in the low-noise case (Fig. 4), visual discrimination between the two orbits is difficult in the medium-noise and high-noise cases (Figs. 5 and 6).

Table I shows the $p$-values calculated by our method for all orbits. In statistical tests, a significance 
(a)

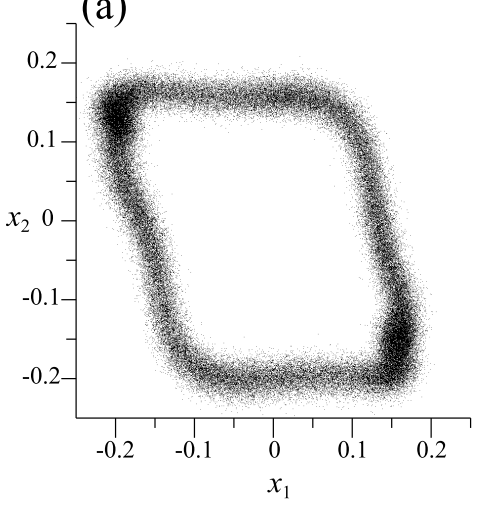

(b)

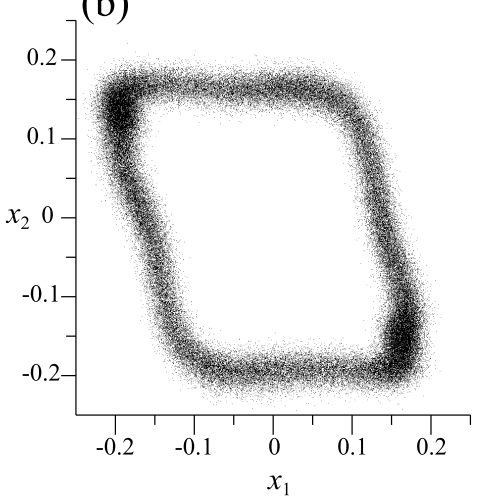

Fig. 6. Orbits of chaotic neural networks in the high-noise case $(\sigma=0.01)$. (a) Quasiperiodic orbit $(\gamma=4.98)$. (b) Periodic orbit $(\gamma=4.9895)$. The other parameters are fixed at $k=0.7, \alpha=1, \beta=2 / 3, d=0.3, \epsilon=0.05$, and $\lambda=-0.9$.

level is typically chosen to be 0.01 or 0.05 . As mentioned in the previous section, quasiperiodicity is accepted if the $p$-value is greater than the significance level in contrast to normal statistical tests. Therefore, to strictly evaluate the $p$-values, we here choose 0.05 as the significance level. In the noiseless case, quasiperiodicity is rejected even if the orbit is quasiperiodic. As mentioned in Sec. 2, our method is constructed on the premise of the presence of dynamical noise. If there is no dynamical noise, the random walk does not occur. Therefore, our method cannot apply to noiseless systems. However, as shown in the low-noise case, our method can correctly discriminate the quasiperiodic and periodic orbits even if the noise intensity is weak. In the medium-noise case, especially, quasiperiodicity is correctly detected by our method even if it is difficult to visually discriminate the quasiperiodic and periodic orbits as shown in Fig. 5 .

Table I. $p$-value of each orbit. The number of data is $M=10,000$. The center of phase is $C=(0,0)$. Bold implies that quasiperiodicity is accepted in the significance level 0.05 . " $<0.01$ " implies that the $p$-value is smaller than 0.01 .

\begin{tabular}{lcccc}
\hline & Noiseless & Low-noise & Medium-noise & High-noise \\
& $\sigma=0$ & $\sigma=0.0001$ & $\sigma=0.001$ & $\sigma=0.01$ \\
& (Fig. 2) & (Fig. 4) & (Fig. 5) & (Fig. 6) \\
\hline Quasiperiodic orbit (a) & $<0.01$ & $\mathbf{0 . 4 5 7}$ & $\mathbf{0 . 1 4 9}$ & $\mathbf{0 . 6 3 5}$ \\
\hline Periodic orbit (b) & $<0.01$ & $<0.01$ & $<0.01$ & $\mathbf{0 . 5 0 5}$ \\
\hline
\end{tabular}

Unfortunately, in the high-noise case, quasiperiodicity is incorrectly accepted even if the orbit is periodic. This is due to noise intensity that exceeds the basin of attraction of the periodic points. We show the structure of the periodic orbit in Fig. 7. The periodic points are separated by the saddle points on the invariant closed curve. Since the attraction on the invariant closed curve is weaker than that of the inside and outside of the curve, the orbit is distributed along the curve in the presence of dynamical noise (see Fig. 5(b)). If the noise intensity is sufficiently low, the orbit remains within the saddle boundaries, which is the situation that we assumed. However, if the tail of the distribution touches the saddle points, the transition to the other periodic point occurs in the orbit. Let $P_{1}, P_{2}, \ldots, P_{15}$ be the 15 periodic points. The subscripts of $P$ imply the sequential order of points along the invariant closed curve. In the noiseless case, since the rotation number of the periodic orbit is $\omega=2 / 15$, the regular transition occurs as follows,

$$
P_{1} \rightarrow P_{3} \rightarrow \cdots P_{15} \rightarrow P_{2} \rightarrow P_{4} \rightarrow \cdots \rightarrow P_{14} \rightarrow P_{1} \rightarrow \cdots
$$

Namely, the regular transition is the order of $P_{i} \rightarrow P_{i+2}$. In the high-noise case, if the noise at $P_{i}$ touches the saddle point between $P_{i}$ and $P_{i+1}$, the transition $P_{i} \rightarrow P_{i+3}$ occurs instead of $P_{i} \rightarrow P_{i+2}$. In the same way, the transition $P_{i} \rightarrow P_{i+1}$ occurs for the saddle point between $P_{i-1}$ and $P_{i}$. Since these irregular transitions randomly occur, the behavior of the orbit is not distinguishable from a 


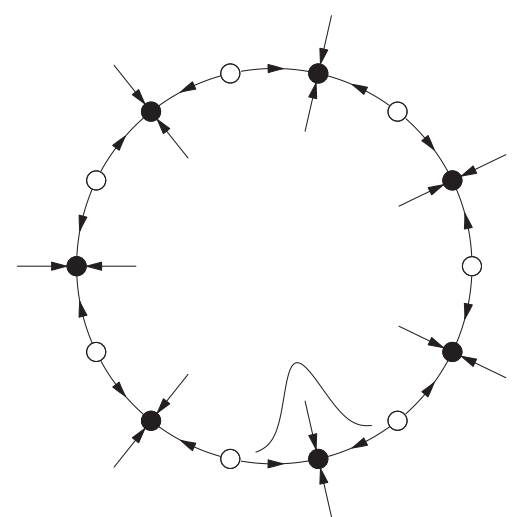

Fig. 7. Structure of periodic orbit (7 period case). Black and white dots are the periodic and saddle points, respectively. In the presence of dynamical noise, the orbit is fluctuated around the periodic points as shown as normal distribution in the curve.

random walk. Therefore, it is necessary for our method that the noise intensity does not frequently exceed the saddle boundaries.

We use three types of the noise intensity, $\sigma=0.0001,0.001$, and 0.01 in the low-noise, mediumnoise, and high-noise cases, respectively. In order to consider noise in circuit experiments, we introduce signal-to-noise ratio (SNR):

$$
\mathrm{SNR}=20 \log _{10} \frac{\sigma_{s}}{\sigma}[\mathrm{dB}],
$$

where $\sigma_{s}$ is the standard deviation of the signal and $\sigma_{s}=0.14$ in our simulation. Then, each intensity is converted to 63 [dB] (low-noise), 43 [dB] (medium-noise), or 23 [dB] (high-noise). Analog circuits are designed to achieve given functional (operating) accuracy, which imposes a minimum allowable noise level. The typical case is 8-bit effective (binary equivalent) circuit implementation accuracy, in which SNR should be larger than $54[\mathrm{~dB}]$ for proper circuit operations. Therefore, our method is applicable to typical analog circuits because the allowable SNR is included between the low-noise and medium-noise cases. Since the medium-noise case corresponds to the circuits with 7-bit effective accuracy (48 [dB] in SNR), the situation in which visual discrimination is difficult as shown in Fig. 5 occurs enough in analog circuit design with various sources of noises such as thermal noise, flicker (1/f) noise, and coupling noise. If the circuits have larger noise (e.g., 36 or 42 [dB] in SNR, which corresponds to the tolerable SNR of a circuit with effective accuracy of 5-bit or 6-bit, respectively), our method may fail to detect quasiperiodicity because the SNR is comparable to the high-noise case. However, such a SNR is allowed only in the design in which accuracy is not a concern.

On the other hand, as mentioned above, our method is not applicable to the noiseless case. We estimate the lower bound of the noise intensity at $\sigma=10^{-6}$. In this case, the SNR is $103[\mathrm{~dB}]$, which is comparable to analog circuits with 16-bit effective accuracy (102 [dB]). The circuits with 16-bit effective accuracy require a special concern to reduce noise as well as circuit/device errors through careful and skillful design and implementation. Therefore, our method is also applicable to the high-accuracy analog circuits in which noise is highly reduced.

We have applied our method to circuit experiments of the chaotic neural networks in the companion paper [15]. In the circuit of the chaotic neural networks, a switched-capacitor implementation is used [30]. There are various uncontrollable subjects associated with circuit implementation such as the shape of the output function that is necessary to design the feedback gain. Nevertheless, the circuit can generate quasiperiodic orbits by using the control method of pole assignment. Our method is applied to the quasiperiodic and periodic orbits generated by the circuit and can correctly discriminate them (see [14] and [15] for details). The circuit design is performed in 8-bit effective accuracy, and hence the results are included in the applicable region of our method. Therefore, our method is applicable to real-world systems represented by implemented circuits. 


\section{Conclusions}

We have proposed a statistical method to test whether a time series is quasiperiodic or not. Our method is based on the equivalence between the noisy irrational rotation and the random walk. It is an important feature of this work that dynamical noise serves as the effective probe to detect quasiperiodicity. In the condition of appropriate intensity of noise, we have shown that our method can correctly discriminate quasiperiodic and periodic orbits. Further work is required to extend our method to the discrimination between quasiperiodicity and chaos.

\section{Acknowledgments}

We thank Professor Motomasa Komuro for his valuable suggestion of the control of quasiperiodicity. This research was supported by the Aihara Project, the FIRST program from JSPS, initiated by CSTP. This work was partially supported by JSPS KAKENHI Grant Number 15KT0074.

\section{References}

[1] S. Das, C.B. Dock, Y. Saiki, M. Salgado-Flores, E. Sander, J. Wu, and J.A. Yorke, "Measuring quasiperiodicity," EPL (Europhysics Letters), vol. 114, no. 4, p. 40005, 2016.

[2] K. Nakano, "Associatron-a model of associative memory," IEEE Transactions on Systems, Man, and Cybernetics, vol. SMC-2, pp. 380-388, July 1972.

[3] J.A. Anderson, "A simple neural network generating an interactive memory," Mathematical Biosciences, vol. 14, no. 3, pp. 197-220, 1972.

[4] T. Kohonen, "Correlation matrix memories," IEEE Transactions on Computers, vol. C-21, pp. 353-359, April 1972.

[5] S. Amari, "Learning patterns and pattern sequences by self-organizing nets of threshold elements," IEEE Transactions on Computers, vol. 21, pp. 1197-1206, November 1972.

[6] H. Nozawa, "A neural network model as a globally coupled map and applications based on chaos," Chaos: An Interdisciplinary Journal of Nonlinear Science, vol. 2, no. 3, pp. 377-386, 1992.

[7] T. Yamada and K. Aihara, "Nonlinear neurodynamics and combinatorial optimization in chaotic neural networks," Journal of Intelligent 83 Fuzzy Systems, vol. 5, no. 1, pp. 53-68, 1997.

[8] M. Hasegawa, T. Ikeguchi, and K. Aihara, "Exponential and chaotic neurodynamical tabu searches for quadratic assignment problems," Control and Cybernetics, vol. 29, no. 3, pp. 773$788,2000$.

[9] Y. Horio, T. Ikeguchi, and K. Aihara, "A mixed analog/digital chaotic neuro-computer system for quadratic assignment problems," Neural Networks, vol. 18, no. 56, pp. 505-513, 2005.

[10] K. Aihara, G. Matsumoto, and Y. Ikegaya, "Periodic and non-periodic responses of a periodically forced Hodgkin-Huxley oscillator," Journal of Theoretical Biology, vol. 109, no. 2, pp. 249-269, 1984.

[11] N. Ichinose and M. Komuro, "Delayed feedback control and phase reduction of unstable quasiperiodic orbits," Chaos: An Interdisciplinary Journal of Nonlinear Science, vol. 24, no. 3, p. 033137, 2014.

[12] K. Aihara, J. Imura, and T. Ueta, eds., Analysis and Control of Complex Dynamical Systems: Robust Bifurcation, Dynamic Attractors, and Network Complexity, ch. 8. Stabilization control of quasiperiodic orbits. Mathematics for Industry, Springer Japan, 2015.

[13] N. Ichinose and M. Komuro, "Multiple delayed feedback control of discrete-time quasi-periodic orbits," IFAC-PapersOnLine, vol. 48, no. 18, pp. 211-214, 2015. 4th IFAC Conference on Analysis and Control of Chaotic Systems CHAOS 2015.

[14] M. Ogawa, Y. Horio, N. Ichinose, and M. Komuro, "Stabilization of unstable quasi-periodic solutions in asymmetric chaotic neural network circuits -numerical simulations and circuit experiments-," Proceedings of International Symposium on Nonlinear Theory and Its Applications, pp. 844-847, 2015. 
[15] Y. Horio, N. Ichinose, and M. Ogawa, "Experimental verification of quasi-periodic-orbit stabilization using a switched-capacitor chaotic neural network circuit," NOLTA, vol. 9, no. 2, pp. 218-230, 2018.

[16] M. Sano and Y. Sawada, "Measurement of the Lyapunov spectrum from a chaotic time series," Physical Review Letters, vol. 55, no. 10, p. 1082, 1985.

[17] C.R. Nelson and C.R. Plosser, "Trends and random walks in macroeconmic time series," Journal of Monetary Economics, vol. 10, no. 2, pp. 139-162, 1982.

[18] C. Granger and P. Newbold, "Spurious regressions in econometrics," Journal of Econometrics, vol. 2, no. 2, pp. 111-120, 1974.

[19] D.A. Dickey and W.A. Fuller, "Distribution of the estimators for autoregressive time series with a unit root," Journal of the American Statistical Association, vol. 74, no. 366, pp. 427-431, 1979.

[20] C. Grebogi, E. Ott, and J.A. Yorke, "Attractors on an N-torus: Quasiperiodicity versus chaos," Physica D Nonlinear Phenomena, vol. 15, pp. 354-373, February 1985.

[21] K. Kamiyama, T. Endo, and M. Komuro, "Bifurcation of 3-torus attractor in certain continuoustime dynamical systems," IFAC-PapersOnLine, vol. 48, no. 18, pp. 122-126, 2015. 4th IFAC Conference on Analysis and Control of Chaotic Systems CHAOS 2015.

[22] M.V. Veldhuizen, "On the numerical approximation of the rotation number," Journal of Computational and Applied Mathematics, vol. 21, no. 2, pp. 203-212, 1988.

[23] R. MacKay, "A simple proof of Denjoy's theorem," Mathematical Proceedings of the Cambridge Philosophical Society, vol. 103, no. 2, pp. 299-303, 1988.

[24] T. Sauer, J.A. Yorke, and M. Casdagli, "Embedology," Journal of Statistical Physics, vol. 65, no. 3, pp. 579-616, 1991.

[25] H. Akhadkulov, M.S.M. Noorani, and A. Dzhalilov, "On invariant measure of the circle maps," Journal of Physics: Conference Series, vol. 435, no. 1, p. 012011, 2013.

[26] E. Brown, J. Moehlis, and P. Holmes, "On the phase reduction and response dynamics of neural oscillator populations," Neural Computation, vol. 16, no. 4, pp. 673-715, 2004.

[27] S.E. Said and D.A. Dickey, "Testing for unit roots in autoregressive-moving average models of unknown order," Biometrika, vol. 71, no. 3, pp. 599-607, 1984.

[28] https://www.r-project.org/.

[29] K. Aihara, T. Takabe, and M. Toyoda, "Chaotic neural networks," Physics Letters A, vol. 144, no. 6, pp. 333-340, 1990.

[30] Y. Horio and K. Suyama, "Switched-capacitor chaotic neuron for chaotic neural networks," 1993 IEEE International Symposium on Circuits and Systems, vol. 2, pp. 1018-1021, May 1993. 